\title{
PET/CT-guided biopsy of liver lesions
}

\author{
Juliano J. Cerci • Franciane Zamparetti Tártari Huber • \\ Mateos Bogoni
}

Received: 30 August 2013/ Accepted: 11 February 2014/Published online: 3 April 2014

(C) Italian Association of Nuclear Medicine and Molecular Imaging 2014

\begin{abstract}
Positron emission tomography/computed tomography $(\mathrm{PET} / \mathrm{CT})$ has had a great impact in oncology; in this field, PET/CT-guided biopsy finds application in the evaluation of liver lesions, among others. The indication for this procedure should be individualized and taken into account the level of suspicion of malignancy and the need for diagnostic confirmation. PET/CT-guided biopsy is feasible and may optimize the diagnostic yield of image-guided interventions.
\end{abstract}

\section{Introduction}

The diagnosis of cancer is a process that begins with a medical history and appropriate physical examination, supplemented by non-invasive diagnostic examinations (imaging and laboratory tests), and ends with histological confirmation.

The liver is a frequent target of metastatic tumors originating in the gastrointestinal tract. It may also be affected secondarily by other primary tumors, such as breast, lung and ovarian cancers [1]. As regards, primary liver cancers, the incidence of hepatocellular carcinoma

Color figures online at http://link.springer.com/article/10.1007/ s40336-014-0056-0.

\section{J. J. Cerci $(\square)$}

Division of PET/CT, Quanta, Diagnóstico Nuclear, R. Almirante Tamadaré, 1000, Curitiba, PR 80045-170, Brazil

e-mail: cercijuliano@hotmail.com

\section{F. Z. T. Huber}

Hospital de Câncer de Barretos, Fundação PIO XII, Barretos, SP, Brazil

\section{Bogoni}

Hospital de Clínicas, Universidade Federal do Paraná, Curitiba, PR, Brazil
(HCC) in the US has been shown to have doubled in relatively recent years [2,3]. However, benign liver lesions may also occur simultaneously in patients with known malignancy or who are under investigation.

With regard to hepatic lesions, differentiation between malignant and benign and also between primary and secondary is necessary to ensure proper treatment in each case. Due to the increased use of high-quality imaging techniques such as multidetector computed tomography (CT) and magnetic resonance imaging (MRI), more smallsize lesions are now being detected.

Over recent decades, positron emission tomography (PET), as a functional imaging technique capable of defining metabolic characteristics of tumor masses, has had a major impact in the oncology field, showing increasing applicability in most of the steps involved in the evaluation patients with cancer (including the initial staging, evaluation of response to treatment and also restaging) [4]. Its advantages include early detection of malignancy-in some cases even before the onset of morphological changes - and characterization of post-treatment lesions (differentiation between malignant tissue and fibrosis) [4-6].

Due to the high sensitivity of this technique in the detection of malignancy, the use of PET in clinical practice has been gaining ground, especially since the introduction of hybrid systems combine PET and conventional CT [7]. The radiopharmaceutical most commonly used in PET is 2- $\left[{ }^{18} \mathrm{~F}\right]$-fluoro2-deoxy-D-glucose (FDG). FDG PET shows higher sensitivity than other non-invasive methods for the detection of hepatic metastases from common tumors such as colorectal, gastric and esophageal cancers [8]. However, even though FDG has given great results in oncological patients, it is not specific to malignant processes. Benign lesions, such as inflammatory processes, are also avid for FDG $[9,10]$. Therefore, percutaneous biopsy may be indicated to determine the real nature of 
FDG uptake in a lesion, especially when the result will affect treatment planning.

In general, PET/CT-guided biopsy is seen as a new-and still evolving - technique, and its value has to be compared with that of other imaging-guided biopsy procedures, already widely used in clinical practice, which include X-ray fluoroscopy, ultrasound scanning (US), CT, and more recently, MRI. $\mathrm{X}$-ray fluoroscopy is a real-time technique widely available for guiding biopsies, but it is typically reserved for large masses that impinge upon fluoroscopically visible structures [11]. US is also a real-time technique, but it is used usually for masses located in the retroperitoneum and deep pelvis, and masses located in the liver, bone or bowel [12]. CT is commonly used to guide abdominal biopsies, but some tumors are not visible without using intravenous contrast material [13] (and when contrast is used its effects are often transient and not longlasting enough to allow visualization of masses throughout the procedure). MRI can also be used; however, open- or widebore magnets are typically needed to reach abdominal masses, and biopsy equipment must be MR compatible [14].

The information provided by these methods is usually anatomical. PET/CT provides both anatomical and metabolic information [7], which naturally makes it a more attractive option, not only for diagnostic imaging but also for refining percutaneous interventions. Most noticeably, it is useful in accessing lesions visible with PET but not with CT, and masses with heterogeneous patterns of FDG uptake (where, on the basis of PET information, the needle can be inserted into the part of a lesion with higher metabolic uptake, which is thus more likely to contain representative cancer cells).

Image-guided biopsy performed using the fine-needle aspiration (FNA) puncture technique or, alternatively, "core biopsy" is a good diagnostic method in patients with focal hepatic lesions, and may be indicated even in small lesions (between 1 and $3 \mathrm{~cm}$ ), both in cirrhotic and in non-cirrhotic individuals [15]. European data from the past decade suggest that in chronic liver disease, $\mathrm{HCC}<2 \mathrm{~cm}$ in diameter accounted for only $<5 \%$ of cases, however recent data from Japanese studies suggest that it may account for $30 \%$ [16-18].

In experienced hands, percutaneous biopsy is a safe, minimally invasive, accurate and cost-effective procedure. However, its accuracy in the evaluation of small hepatic lesions, measuring $2 \mathrm{~cm}$ or less in diameter, is significantly higher with the "core biopsy" (94.4\%) than with the FNA biopsy method (66.8\%) [15].

\section{Indications}

Liver biopsy finds application in several contexts, e.g., for identifying benign nodular hepatocellular lesions, i.e., inflammatory/infectious lesions; or distinguishing macroregenerative lesions, dysplastic nodules, focal nodular hyperplasia, and liver cell adenoma from reactive hepatocytes. It may also be applied to distinguish well-differentiated HCC from benign hepatocellular nodular lesions, or poorly differentiated HCC from cholangiocarcinoma and metastatic carcinomas, and to determine the histogenesis of malignant tumor and the primary site of the malignant tumor of origin [19].

PET/CT-guided biopsy of the liver is especially useful in lesions seen on PET scans, but not detected by other methods such as CT [20, 21], and in masses with a heterogeneous pattern of glucose uptake seen on FDG PET imaging. In these latter cases, PET/CT allows the physician performing the biopsy to access the region with the highest FDG uptake, i.e., the region more likely to contain representative cancer cells $[20,22]$; in the same way, the technique is useful in lymph node chains where only some lymph nodes present FDG uptake.

\section{Contraindications}

Contraindications to percutaneous liver biopsy guided by PET/CT are reported to be the same as those to CT-guided or percutaneous blind liver biopsy, namely, lack of a safe access route, uncorrectable bleeding diathesis for biopsy, and uncooperative patients [23]. However, the last two can be considered relative contraindications.

In the case of uncooperative patients, light sedation during the guided biopsy procedure might be recommended, as this would reduce the patient's mobility and, thus, the risk of complications [21].

\section{Pre-procedure care/preparing}

As with all procedures, even if minimally invasive, the patient should be informed beforehand about how it will be performed and its inherent risks.

Since bleeding could be a major complication of the procedure, another important aspect, to reduce this risk, is to suspend medications that alter blood clotting, such as aspirin, heparin, and so on, at least 7-10 days before the procedure.

Before the biopsy, the patient should also undergo laboratory tests such as complete blood count, including platelet count, and coagulation studies. Biopsies are contraindicated in the presence of platelet counts $<100,000 / \mu 1$, altered coagulation parameters, or any other medical contraindication to the procedure [20].

\section{Description of the technique}

\section{FDG PET/CT}

Whole body PET/CT images are acquired 60-90 min after intravenous administration of $296-444 \mathrm{MBq}(8-12 \mathrm{mCi})$ 
of FDG, and FDG PET findings should be interpreted by an experienced nuclear medicine physician. After patients have undergone FDG PET/CT scanning to define the site of biopsy, the course to be followed by the needle is also evaluated, considering the position of the suspicious lesion and its relation to anatomical structures.

\section{Biopsy procedure}

Patients are positioned and immobilized according to the location of the lesion and the chosen biopsy approach. After completion of asepsis and antisepsis, local anesthesia is performed. As mentioned above, light sedation might be required in the case of non-cooperative patients.

A suitable coaxial needle is inserted in the point previously identified. The "core biopsy" technique should be preferred, as the histopathological analysis is more accurate than the cytological evaluation permitted by FNA. The angle and direction of the needle are adjusted according to the position of the suspicious lesion under the guidance of CT fluoroscopic imaging. After placing the point of the coaxial needle in the border of the suspicious lesion, one bed position of default images of PET/ CT can be acquired to confirm the correct position of the coaxial needle.

The core of the coaxial needle is pulled out and a semiautomatic biopsy needle is inserted. The puncture is confirmed as satisfactory under fluoroscopy and the biopsy site is recorded (if the lesions were small or difficult to differentiate from important structures such as peripheral vessels, enhanced CT could be used to optimize the visualization of different anatomical structures, after which then specimen is cut and drawn at 1 or $2 \mathrm{~cm}$ stall of biopsy needle).

Three or four specimens are collected. After removal of the needle, manual compression should be performed for 2-5 min at the puncture site. The specimens are fixed in $10 \%$ formalin and sent for histopathological examination. Intraoperative histopathological analysis, with a frozen section, might be useful as it could reduce the need to perform new biopsies due to unreliable results.

Patients are observed for at least $3 \mathrm{~h}$ after the procedure to ensure hemodynamic stability and monitoring of their respiratory condition. CT might be performed $3 \mathrm{~h}$ after the biopsy in patients in whom the needle transfixes the pleura, liver and stomach wall to perform the biopsy procedure.

\section{Risk of complications}

Bleeding is the most common complication of PET/CTguided biopsy of lesions in the liver and is a risk intrinsic to the biopsy procedure. Death has also been reported in a very small number of patients.
Bleeding is considered minor when only supportive and observational treatment is required. Minor bleeding occurs in about 10-20\% of patients. Major bleeding is instead defined as bleeding requiring interventions such as surgery and transfusion, and it occurs in about $1-5 \%$ of patients. Mortality is reported to be below $1 \%$ of cases [24-26]. These complications are often linked to vascularity and location of lesions, as well as needle size [27].

Another growing concern is the possibility of seeding of tumor cells through the needle paths. The impact of this phenomenon varies according to the diameter of the needle, the number of needle passages, and the amount of parenchyma surrounding the lesion to be traversed by the needle [28], and it ranges from $1.6 \%$ in biopsies of HCC [29] to 16-19\% in biopsies of liver metastases from colorectal cancer [30, 31]. However, additional studies are needed.

\section{Relevance}

The liver is a common site of metastases, but it is also associated with an increasing incidence of primary cancer, making it an important organ for oncological evaluation in all age groups [32-34]. Due to the high incidence of benign hepatic lesions (20\%), characterization of lesions is crucial [35].

Histopathological confirmation of benign focal liver lesions is important to avoid surgery that is, frequently, unnecessary. Meanwhile, the diagnosis of a malignant lesion of the liver often leads to decisions to undertake more aggressive therapies such as surgical resection, liver transplantation, percutaneous ablative therapies or systemic chemotherapy [36-38].

Levels of sensitivity and specificity for the detection and characterization of focal liver lesions have improved substantially due to the advances in imaging techniques [39]. However, the specificity in the characterization of small lesions is not high enough to allow reliable diagnosis of benignity or malignancy [40-43]. This problem is especially pronounced in cirrhotic liver parenchyma because of the heterogeneous and nodular morphology [43, 44]. Functional imaging studies such as FDG PET/CT may assist in the diagnosis, although they are known to be limited in the detection and characterization of lesions $0.6-0.8 \mathrm{~cm}$ or smaller [45].

Dietlein et al. [46] performed a systematic review, which included 15 studies that had at least 35 patients with suspected recurrent colorectal cancer or colorectal liver metastases. Five studies including 445 patients were used to create pooled estimates of diagnostic accuracy in restaging. PET showed a sensitivity and specificity (with $95 \%$ CIs) of $94 \%(91-96 \%)$ and $78 \%$ (69-86\%), while 


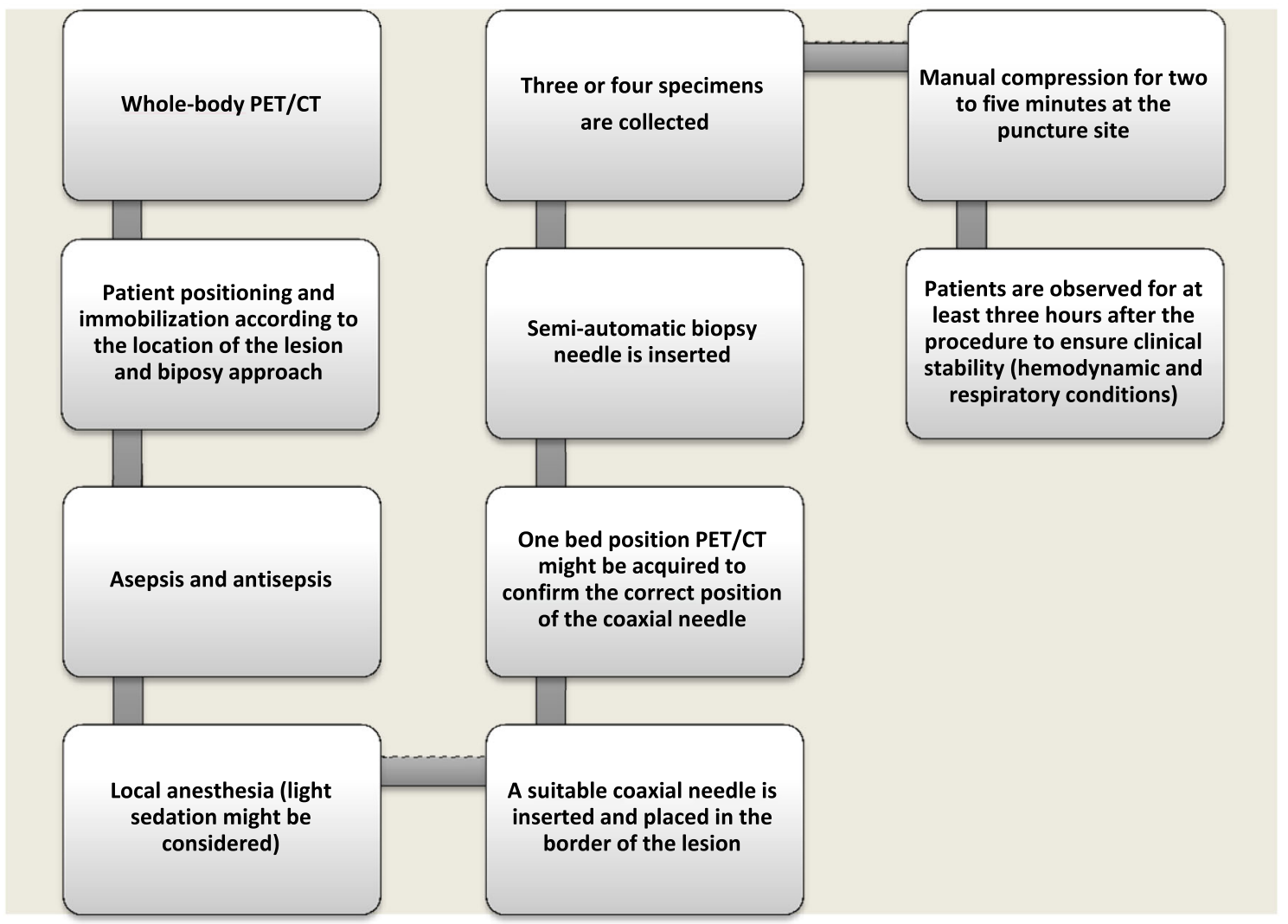

Fig. 1 PET/CT-guided biopsy: technical procedure diagram (color figure online)

for CT the sensitivity and specificity were $73 \%(68-78 \%)$ and $62 \%(52-72 \%)$. The summarized results of ten studies (741 patients) provided evidence of change in patient management: PET correctly changed staging in $27 \%$ of patients; $4 \%$ had their staging changed incorrectly as a result of PET.

Given that FDG PET-positive results can define conduct, determining institution of therapy (be it surgery, chemotherapy or radiotherapy) and influencing the risk of morbidity and mortality, it is recommended, whenever possible, to confirm the findings by anatomical pathology studies before treatment. The trend of biopsies in focal liver lesions indicates that the incidence of focal small lesions is increasing, but imaging techniques are frequently inconclusive in determining the nature of these lesions.

Nguyen et al. [47] evaluated 227 patients who underwent imaging-guided biopsy of 231 PET-positive extrapulmonary lesions. Bone (96\%) and liver (90\%) lesions had significantly higher positive predictive values (PPVs) for malignancy compared with other locations, whereas lymph nodes $(60 \%)$ had a significantly lower PPV for malignancy (Fig. 1).
The high rates of technical success in acquiring samples sufficient for histopathological analysis in general biopsies guided by PET/CT $(98.5 \%)$, the resulting improved definition of benign and malignant histopathological findings, and the low complication rates, all suggest that liver biopsies guided by PET/CT have a promising future, in terms of indications and performance, even though further studies are required [21]. Our group found that FDG PET improved the selection of biopsy sites. Twenty-three out of $130(17.7 \%)$ patients were referred for PET/CT-guided biopsy due to previous non-tumoral biopsy results, and 21 were found to have malignant lesions [21]. Metabolic characterization might also be useful for biopsy site selection, especially in large lesions with necrotic areas (Figs. 2, 3), and the same applies to lesions with no anatomical reference.

An update of our group's results is as follows: of 217 PET/CT-guided biopsies performed, 36 were liver biopsies (Table 1). Two lesions were re-accessed due to unreliable histological results. Histology showed that 27/36 (75\%) were oncological lesions, while 9/36 (25\%) lesions were benign (inflammatory cells or necrotic tissue). There was 


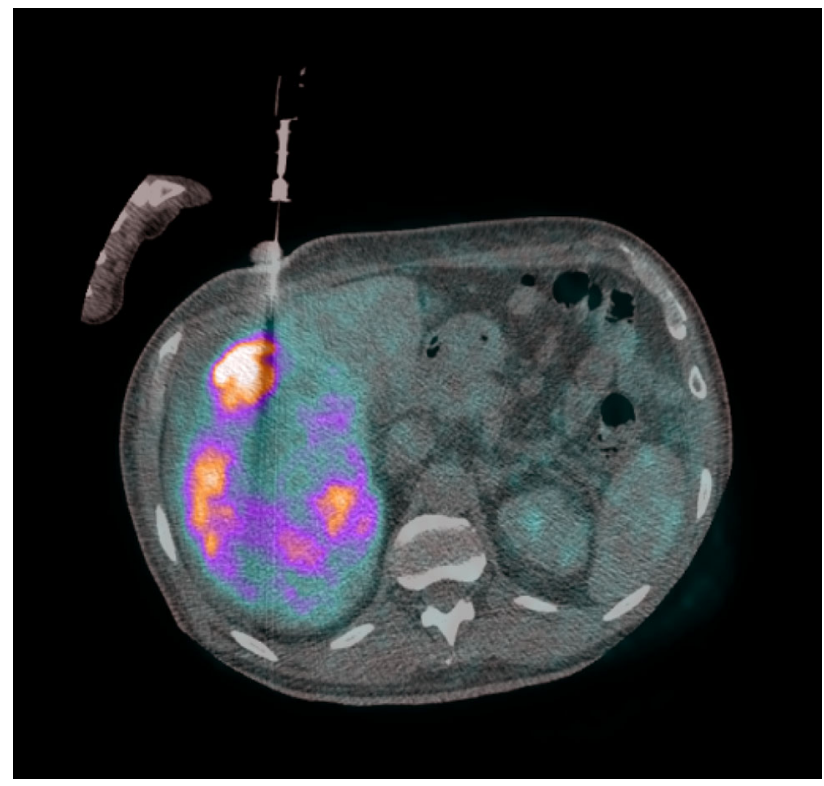

Fig. 2 Evaluation of a large liver lesion in a 46-year-old male patient, with a previous non-tumor biopsy result. FDG PET showed a heterogeneous mass and guided the needle to a highly metabolic area inside the lesion. Histology confirmed a hepatocellular carcinoma (color figure online)

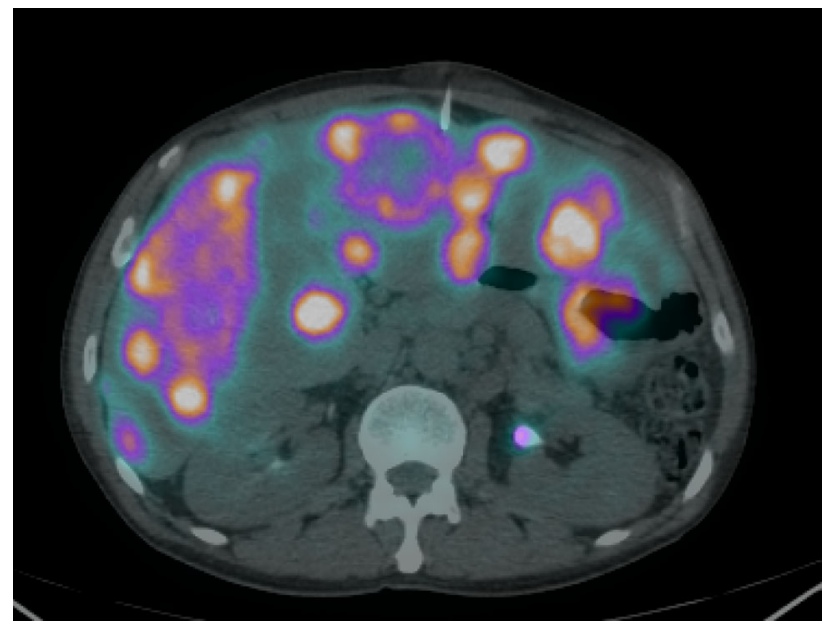

Fig. 3 A 67-year-old male patient with multiple nodules and abdominal pain. FDG PET showed a central lesion in the left lung, mediastinal lymph nodes and multiple hepatic lesions. The larger liver lesion presented heterogeneous uptake. The needle was directed to a highly metabolic area within the lesion. Histology confirmed an adenocarcinoma (color figure online)

no significant difference between SUV of malignant and benign lesions; SUV of malignant and benign lesions ranged from 3.5 to 27.6 (median $9.1 \pm 6.6$.); and 4.2 to 18.6 (median $9.1 \pm 4.4$ ), respectively ( $p 0.7$ ).

In the liver biopsies, the FDG PET was considered critical for performing the biopsy in $11 / 36$ patients $(30.5 \%)$. Clinical complications included minor bleeding (requiring only supportive and observational treatment),
Table 1 Clinical characteristics of the patients submitted to liver biopsy

\begin{tabular}{|c|c|c|c|c|c|}
\hline & Gender & Age & SUV & Histology & $\begin{array}{l}\text { CT correspondent } \\
\text { lesion }\end{array}$ \\
\hline 1 & Male & 67 & 4.3 & Malignant & No \\
\hline 2 & Male & 78 & 10.5 & Malignant & Yes \\
\hline 3 & Male & 69 & 11.1 & Benign & No \\
\hline 4 & Female & 65 & 9.8 & Malignant & Yes \\
\hline 5 & Male & 60 & 4.8 & Benign & Yes \\
\hline 6 & Male & 69 & 27.6 & Malignant & Yes \\
\hline 7 & Female & 59 & 8.8 & Benign & No \\
\hline 8 & Male & 60 & 11.1 & Malignant & Yes \\
\hline 9 & Female & 57 & 8.2 & Malignant & Yes \\
\hline 10 & Male & 78 & 15.3 & Malignant & Yes \\
\hline 11 & Female & 60 & 4.2 & Benign & Yes \\
\hline 12 & Male & 71 & 12.7 & Malignant & Yes \\
\hline 13 & Male & 65 & 14.7 & Malignant & No \\
\hline 14 & Female & 72 & 4.6 & Malignant & Yes \\
\hline 15 & Male & 67 & 10.3 & Malignant & Yes \\
\hline 16 & Male & 64 & 11.7 & Benign & Yes \\
\hline 17 & Male & 44 & 25.4 & Malignant & Yes \\
\hline 18 & Female & 68 & 17.7 & Malignant & Yes \\
\hline 19 & Female & 51 & 10.2 & Malignant & Yes \\
\hline 20 & Male & 51 & 8.5 & Malignant & Yes \\
\hline 21 & Female & 82 & 10.3 & Malignant & Yes \\
\hline 22 & Female & 43 & 15.5 & Malignant & Yes \\
\hline 23 & Female & 47 & 18.6 & Benign & Yes \\
\hline 24 & Female & 51 & 7.7 & Malignant & Yes \\
\hline 25 & Female & 68 & 24.6 & Malignant & Yes \\
\hline 26 & Female & 59 & 10.8 & Malignant & Yes \\
\hline 27 & Male & 75 & 9.1 & Benign & No \\
\hline 28 & Male & 54 & 4.3 & Malignant & No \\
\hline 29 & Male & 54 & 4.3 & Malignant & No \\
\hline 30 & Male & 30 & 6.7 & Benign & No \\
\hline 31 & Male & 30 & 6.7 & Benign & No \\
\hline 32 & Male & 77 & 12.4 & Malignant & Yes \\
\hline 33 & Female & 62 & 9.5 & Malignant & No \\
\hline 34 & Male & 40 & 3.5 & Malignant & $?$ \\
\hline 35 & Female & 61 & 7.4 & Malignant & No \\
\hline 36 & Female & 55 & 4.9 & Malignant & Yes \\
\hline
\end{tabular}

which occurred in 4/36 (11.1\%) patients; one major bleeding episode requiring transfusion occurred in one patient with an angiosarcoma lesion. There was no mortality related to the procedure in our series.

Finally, percutaneous biopsy could have been performed by fusing prior PET/CT scan images with conventional CT scan images obtained during the procedure [20]. Even though this technique is also relatively new, and experience is limited, preliminary studies, performing biopsies in several body sites, have shown it to be highly beneficial for achieving a definite diagnosis [48]. Radiation exposure to 
patients undergoing whole body PET/CT ranges between 8.5 and $32.1 \mathrm{mSv}[49,50]$. The CT component generally contributes $54-81 \%$ of the total combined dose [50]. During a PET/CT-guided biopsy, patients are exposed to radiation from both the initial diagnostic PET/CT scan and the additional CT scans obtained during the biopsy [50]. However, these patients would have undergone a diagnostic PET/CT scan regardless of the biopsy. Therefore, the additional radiation imparted to patients as a result of the biopsy derives only from the short CT scans acquired during needle placement.

Besides its application in biopsies, FDG PET/CT also might be useful for guiding treatment, such as percutaneous ablation, allowing evaluation at completion of the ablation, to assess ablation adequacy [51]. The pathological condition found in the tumor was metastatic colorectal adenocarcinoma in 18 lesions, primary HCC in one lesion, and a variety of metastatic tumors in the remaining ten lesions. A total of $4 \mathrm{mCi}(148 \mathrm{MBq})$ of FDG was administered before the procedure for localization and imaging guidance. At completion of the ablation, an additional $8 \mathrm{mCi}(296 \mathrm{MBq}$ ) of FDG was administered to assess ablation adequacy. Results of subsequent imaging follow-up were used to determine whether post-ablation imaging after the second dose of FDG reliably helped in predicting the complete tumor ablation.

As the application of FDG PET in the workup of oncological patients grows, it is natural to expect PET/CT-guided intervention to gain ground at the same time, especially given its contribution to the detection of metabolic features of malignancy early on, even before morphological changes appear. Furthermore, the growing interest in accessing masses with PET/CT guidance is also due to its ability to determine, within a lesion, the most accessible region showing higher metabolic uptake. Naturally, the physician responsible for the biopsy must have an excellent understanding of the FDG uptake characteristics related to malignant processes to define what, of all the metabolic information provided by FDG PET, is truly reliable [22].

Conflict of interest Juliano J. Cerci, Franciane Zamparetti Tártari Huber, Mateos Bogoni have no conflicts of interest to declare.

Ethical statement Human and Animal Studies to the effect that all human studies have been approved by the appropriate ethical committee, and have therefore been performed in accordance with the pertinent ethical guidelines.

\section{References}

1. Weiss L, Bronk J, Pickren JW, Lane WW (1981) Metastatic patterns and target organ arterial blood flow. Invasion Metastasis $1: 126-135$

2. Ambrus JL, Ambrus CM, Mink IB, Pickren JW (1975) Causes of death in cancer patients. J Med 6:61-64
3. El-Serag HB, Mason AC (1999) Rising incidence of hepatocellular carcinoma in the United States. N Engl J Med 340:745-750

4. Pauwels EK, Ribeiro MJ, Stoot JH, McCready VR, Bourguignon M, Mazière B (1998) FDG accumulation and tumor biology. Nucl Med Biol 25(4):317-332

5. Bomanji JB, Costa DC, Ell PJ (2001) Clinical role of positron emission tomography in oncology. Lancet Oncol 2(3):157-164

6. Hustinx R, Bénard F, Alavi A (2002) Whole-body FDG-PET imaging in the management of patients with cancer. Semin Nucl Med 32(1):35-46

7. Beyer T, Townsend DW, Brun T et al (2000) A combined PET/ CT scanner for clinical oncology. J Nucl Med 41(8):1369-1379

8. Kinkel K, Lu Y, Both M, Warren RS, Thoeni RF (2002) Detection of hepatic metastases from cancers of the gastrointestinal tract by using noninvasive imaging methods (US, CT, MR imaging, PET): a meta-analysis. Radiology 224:748-756

9. Zhuang H, Alavi A (2002) 18-Fluorodeoxyglucose positron emission tomographic imaging in the detection and monitoring of infection and inflammation. Semin Nucl Med 32(1):47-59

10. El-Haddad G, Alavi A, Mavi A, Bural G, Zhuang H (2004) Normal variants in $\left[{ }^{18} \mathrm{~F}\right]$-fluorodeoxyglucose PET imaging. Radiol Clin N Am 42(6):1063-1081viii

11. Pereiras RV, Meiers W, Kunhardt B et al (1978) Fluoroscopically guided thin needle aspiration biopsy of the abdomen and retroperitoneum. AJR Am J Roentgenol 131:197-202

12. Rasmussen SN, Holm HH, Kristensen JK, Barlebo H (1972) Ultrasonically-guided liver biopsy. Br Med J 2:500-502

13. Ferrucci JT Jr, Wittenberg J (1978) CT biopsy of abdominal tumors: aids for lesion localization. Radiology 129:739-744

14. Silverman SG, Collick BD, Figueira MR et al (1995) Interactive MR-guided biopsy in an open-configuration MR imaging system. Radiology 197:175-181

15. Ma X, Arellano RS, Gervais DA, Hahn PF, Mueller PR, Sahani DV (2010) Success of image-guided biopsy for small $(\leq 3 \mathrm{~cm})$ focal liver lesions in cirrhotic and noncirrhotic individuals. J Vasc Interv Radiol 21:1539-1547

16. Ikai I, Arii S, Kojiro M et al (2004) Reevaluation of prognostic factors for survival after liver resection in patients with hepatocellular carcinoma in a Japanese nationwide survey. Cancer 101:796-802

17. Bolondi L, Gaiani S, Celli N et al (2005) Characterization of small nodules in cirrhosis by assessment of vascularity: the problem of hypovascular hepatocellular carcinoma. Hepatology 42:27-34

18. Kojiro M (2004) Focus on dysplastic nodules and early hepatocellular carcinoma: an eastern point of view. Liver Transpl 10:S3-S8

19. Wee A (2005) Fine needle aspiration biopsy of the liver: algorithmic approach and current issues in the diagnosis of hepatocellular carcinoma. Cytojournal 2:7

20. Tatli S, Gerbaudo VH, Mamede M, Tuncali K, Shyn PB, Silverman SG (2010) Abdominal masses sampled at PET/CT-guided percutaneous biopsy: initial experience with registration of prior PET/CT images. Radiology 256:305-311

21. Cerci JJ, Pereira Neto CC, Krauzer C, Sakamoto DG, Vitola JV (2013) The impact of coaxial core biopsy guided by FDG PET/ $\mathrm{CT}$ in oncological patients. Eur $\mathrm{J}$ Nucl Med Mol Imaging 40(1):98-103

22. Kobayashi K, Bhargava P, Raja S, Nasseri F, Al-Balas HA, Smith DD, George SP, Vij MS (2012) Image-guided biopsy: what the interventional radiologist needs to know about PET/CT. Radiographics 32(5):1483-501

23. Chhieng DC (2004) Fine needle aspiration biopsy of liver-an update. World J Surg Oncol 2:5

24. Buscarini L, Fornari F, Bolondi L, Colombo P, Livraghi T, Magnolfi F, Rapaccini GL, Salmi A (1990) Ultrasound-guided 
fine-needle biopsy of focal liver lesions: techniques, diagnostic accuracy and complications. A retrospective study on 2091 biopsies. J Hepatol 11:344-348

25. Chawla YK, Ramesh GN, Kaur U, Bambery P, Dilawari JB (1990) Percutaneous liver biopsy: a safe outpatient procedure. J Gastroenterol Hepatol 5:94-95

26. Smith EH (1991) Complications of percutaneous abdominal fineneedle biopsy. Review. Radiology 178:253-258

27. Pitman MB (1998) Fine needle aspiration biopsy of the liver. Principal diagnostic challenges. Clin Lab Med 18:483-506

28. Michielsen PP, Duysburgh IK, Francque SM, Van der Planken M, Van Marck EA, Pelckmans PA (1998) Ultrasonically guided fine needle puncture of focal liver lesions. Review and personal experience. Acta Gastroenterol Belg 61:158-163

29. Durand F, Regimbeau JM, Belghiti J et al (2001) Assessment of the benefits and risks of percutaneous biopsy before surgical resection of hepatocellular carcinoma. J Hepatol 35:254-258

30. Jones OM, Rees M, John TG, Bygrave S, Plant G (2005) Biopsy of resectable colorectal liver metastases causes tumour dissemination and adversely affects survival after liver resection. $\mathrm{Br} \mathbf{J}$ Surg 92:1165-1168

31. Rodgers MS, Collinson R, Desai S, Stubbs RS, McCall JL (2003) Risk of dissemination with biopsy of colorectal liver metastases. Dis Colon Rectum 46:454-458 (discussion 458-459)

32. Bosch FX, Ribes J, Diaz M, Cleries R (2004) Primary liver cancer: worldwide incidence and trends. Gastroenterology 127:S5-S16

33. El-Serag HB, Davila JA, Petersen NJ, McGlynn KA (2003) The continuing increase in the incidence of hepatocellular carcinoma in the United States: an update. Ann Intern Med 139:817-823

34. Shea KA, Fleming LE, Wilkinson JD, Wohler-Torres B, McKinnon JA (2001) Hepatocellular carcinoma incidence in Florida. Ethnic and racial distribution. Cancer 91:1046-1051

35. Martin DR, Semelka RC (2001) Imaging of benign and malignant focal liver lesions. Magn Reson Imaging Clin N Am 9:785-802, vi-vii

36. Kammula US, Buell JF, Labow DM, Rosen S, Millis JM, Posner MC (2001) Surgical management of benign tumors of the liver. Int J Gastrointest Cancer 30:141-146

37. Bruix J, Sherman M, Llovet JM et al (2001) Clinical management of hepatocellular carcinoma. Conclusions of the Barcelona-2000 EASL conference. European Association for the Study of the Liver. J Hepatol 35:421-430

38. Vogl TJ, Muller PK, Mack MG, Straub R, Engelmann K, Neuhaus P (1999) Liver metastases: interventional therapeutic techniques and results, state of the art. Eur Radiol 9:675-684

39. Kanematsu M, Hoshi H, Imaeda $T$ et al (1997) Detection and characterization of hepatic tumors: value of combined helical CT hepatic arteriography and CT during arterial portography. AJR Am J Roentgenol 168:1193-1198

40. Peterson MS, Baron RL, Dodd GD 3rd et al (1992) Hepatic parenchymal perfusion defects detected with CTAP: imagingpathologic correlation. Radiology 185:149-155

41. Yu JS, Kim KW, Sung KB, Lee JT, Yoo HS (1997) Small arterial-portal venous shunts: a cause of pseudolesions at hepatic imaging. Radiology 203:737-742

42. Lim JH, Cho JM, Kim EY, Park CK (2000) Dysplastic nodules in liver cirrhosis: evaluation of hemodynamics with CT during arterial portography and CT hepatic arteriography. Radiology 214:869-874

43. Horigome H, Nomura T, Saso K, Itoh M, Joh T, Ohara H (1999) Limitations of imaging diagnosis for small hepatocellular carcinoma: comparison with histological findings. J Gastroenterol Hepatol 14:559-565

44. Kanematsu M, Hoshi H, Yamada T et al (1999) Small hepatic nodules in cirrhosis: ultrasonographic, $\mathrm{CT}$, and MR imaging findings. Abdom Imaging 24:47-55

45. Frohlich A, Diederichs CG, Staib L, Vogel J, Beger HG, Reske SN (1999) Detection of liver metastases from pancreatic cancer using FDG PET. J Nucl Med 40:250-255

46. Dietlein M, Weber W, Schwaiger M, Schicha H (2003) ${ }^{18} \mathrm{~F}$ Fluorodeoxyglucose positron emission tomography in restaging of colorectal cancer. Nuklearmedizin 42(4):145-156

47. Nguyen ML, Gervais DA, Blake MA, Mueller PR, Sahani DV, Hahn PF, Uppot RN (2013) Imaging-guided biopsy of $\left({ }^{18}\right) \mathrm{F}$ FDG-avid extrapulmonary lesions: do lesion location and morphologic features on CT affect the positive predictive value for malignancy? AJR Am J Roentgenol 201(2):433-8

48. Purandare NC, Kulkarni AV, Kulkarni SS, Roy D, Agrawal A, Shah S, Rangarajan V (2013) ${ }^{18}$ F-FDG PET/CT-directed biopsy: does it offer incremental benefit? Nucl Med Commun 34(3):203-210

49. Brix G, Lechel U, Glatting G et al (2005) Radiation exposure of patients undergoing whole-body dual-modality ${ }^{18}$ F-FDG PET/CT examinations. J Nucl Med 46:608-613

50. Huang B, Law MW, Khong PL (2009) Whole-body PET/CT scanning: estimation of radiation dose and cancer risk. Radiology 251:166-174

51. Ryan ER, Sofocleous CT, Schöder H, Carrasquillo JA, Nehmeh S, Larson SM, Thornton R, Siegelbaum RH, Erinjeri JP, Solomon SB (2013) Split-dose technique for FDG PET/CT-guided percutaneous ablation: a method to facilitate lesion targeting and to provide immediate assessment of treatment effectiveness. Radiology 268(1):288-295 